\title{
A CULTURA DE APRENDIZAGEM TRANSDISCIPLINAR COMO MOTOR PARA IMPULSIONAR A TELESSAÚDE NO PANAMÁ
}

The Culture of Transdisciplinary Learning as an Engine to Drive Telehealth in Panama

\author{
Arlete Pinel'; Víctor López Cabrera²
}

Resumo Entre 2011-2013, foram organizados no Panamá cinco eventos internacionais sequenciais para mostrar a realidade "glocal" (global e local) da telessaúde a profissionais e estudantes dos campos saúde e tecnologia. Nesse período, 110 expertos de 17 países compartilharam seus conhecimentos com o talento panamenho e o país pode retransmitir essa aprendizagem a mais de 2.000 pessoas em 33 países.

Palavras-chave: Telemedicina, Sistemas de Informação em Saúde, Cuidado em Saúde, Saúde Global.

Abstract Between 2011-2013, there were five sequential Panama international events to show the "glocal" reality (global and local) of telehealth to professionals and students of health and technology fields. During this period, 110 experts from 17 countries shared their knowledge with the Panamanian talent and the country could relay that learning to over 2,000 people in 33 countries.

Keywords: Telemedicine, Health Information Systems, Delivery of Healthcare, World Health. 


\section{Introdução}

Panamá mantém um dos maiores índices de crescimento econômico da América Latina e conta com a melhor infraestrutura de telecomunicações da região'. Embora o país tenha a força financeira para impulsar soluções tecnológicas dentro do sistema de saúde, ainda a telessaúde não se consolidou como um sistema integral e integrado, capaz de promover equitativamente a assistência às populações mais vulneráveis e pobres. O sistema público carece de dois perfis importantes: profissionais da saúde que possam rapidamente adaptar-se às novas tecnologias e a uma cultura da colaboração à distância; e profissionais da informática que possam realizar a adequação e transferência tecnológica às necessidades do setor saúde.

\section{Discussão}

Cientes de que um programa nacional de telessaúde precisa de talento humano capacitado no binômio saúde/informática e comprometido com todos os estratos sociais, um grupo de profissionais e 26 instituições nacionais e internacionais ${ }^{[1]}$ impulsaram os seguintes eventos gratuitos entre 2011 e 2013:

- Conferência Internacional de Atualização em Informática Médica e Tecnologia Aplicada à Saúde e ao Desenvolvimento Social; e $1^{\circ}$ Curso Nacional de Introdução à Informática Médica e a Telessaúde (2011)

Objetivo: Oferecer aos profissionais do sistema público de saúde - especialmente aqueles em áreas remotas uma visão ampla da telessaúde como instrumento de desenvolvimento social.

\section{- 1a Conferencia Internacional Sobre Mulheres e as} TIC: Inclusão Digital para a Saúde e o Desenvolvimento: www.mujeresglobales.utp.ac.pa (2012)
Objetivo: Analisar diferentes facetas da exclusão digital vinculada ao gênero que interferem na participação das mulheres nas respostas tecnológicas relacionadas à saúde e ao desenvolvimento sustentável. Durante o evento se lançaram duas iniciativas internacionais: Mulheres Globais: Iniciativa Sul-Sul de Inclusão Digital e WeTelemed - Rede Global de Mulheres em Telemedicina.

\section{- $1^{\circ}$ Congresso Virtual de Inovação Tecnológica para} a Saúde Pública, a Inteligência Epidemiológica e a Vigilância Global: www.congresovirtual2013.utp.ac.pa (2013)

Objetivo: Compartilhar soluções tecnológicas utilizadas para enfrentar as ameaças globais à saúde, melhorar os programas e formar novas mentalidades para o futuro da saúde. A utilização das redes sociais gerou uma participação inesperada de América Latina, África, Europa e Ásia que em alguns momentos superou as mil pessoas assistindo simultaneamente.

\section{- Curso Virtual Introdução à Informática em Saúde e a Telemedicina (2013)}

Objetivo: Promover a telessaúde através da cooperação interinstitucional entre a Faculdade de Medicina da Universidade do Panamá e a Universidade Tecnológica do Panamá na esperança que a experiência conduza à formação conjunta em informática em saúde e telemedicina. O curso foi administrado em colaboração com o Instituto Edumed do Brasil.

Ao entrar em contato com a experiência de 110 especialistas de 17 países (Afeganistão, Argentina, Brasil, Chile, Colômbia, Espanha, Estados Unidos, França, Malásia, Mali, México, Nigéria, Panamá, Reino Unido, Suíça, Taiwan, Venezuela), os profissionais e estudantes panamenhos foram sensibilizados à realidade "glocal" (global e local) da telessaúde. O país pode repassar essa

[1] Universidad Tecnológica de Panamá (UTP), Universidad de Panamá (UP), Secretaría Nacional de Ciencia y Tecnología (SENACYT), Ministerio da Salud (MINSA), Caja de Seguro Social (CSS), Genos Global, Millennia2015 WeHealth, Foundation Dominic, Instituto Edumed, Centro Regional de Capacitación en Salud/ICGES, US Naval Health Research Center, Johns Hopkins University entre outras. 
aprendizagem por várias vias a mais de 2.000 pessoas em 33 países.

O processo revelou que a exposição ao uso ampliado da telessaúde gera interesse e demanda entre os setores. Entretanto, precisa haver um maior compromisso governamental e coordenação interinstitucional para formar competências transdisciplinares que sustentem a modernização do sistema de saúde ${ }^{2-5}$.

\section{Comentários Finais}

A falta de oportunidades de formação e de integração dos profissionais da saúde e tecnologia aumenta a resistência ao uso cotidiano da telessáude. Recomendamos:

- Enfatizar a formação básica: O treinamento após a formação é paliativo. Precisa-se mostrar "o futuro" aos estudantes e jovens profissionais para que se criem competências complementárias que permeiem todos os níveis do sistema de saúde.

- Promover a aprendizagem colaborativa: Precisa haver mais esforços na promoção de redes nacionais e interregionais de aprendizagem, pesquisa e prática entre universidades, serviços e a empresa privada.

\section{- Promover o uso significativo das tecnologias: $O$} foco de atenção precisa mudar das tecnologias às pessoas para que a telessaúde possa ser optimizada.

\section{- Modernizar a infraestrutura de aprendizagem: A} inovação começa pela forma de aprender. Promover mudanças na maneira em que se formam profissionais no meio panamenho resultará em sistemas capazes de utilizar a tecnologia para inovar todas suas funções ${ }^{6}$.
Referências

1. Secretaría Nacional de Ciencia, Tecnología e Innovación de la República de Panamá (SENACYT). Plan Estratégico Nacional de Ciencia, Tecnología e Innovación 2010-2014 (2010).

2. Gomez, L. M., Gomez, K. Gifford, B. G. "Educational Innovation with Technology: A New Look at Scale and Opportunity to Learn." The Aspen Institute Congressional Program Conference Transforming America's Education Through Innovation and Technology (2010)

3. Organización Panamericana de la Salud/Organización Mundial de la Salud. Estrategia y Plan de Acción sobre eSalud. 51 Consejo Directivo, 63a Sesión del Comité Regional, Washington, DC, 26-30 de septiembre (2011)

4. Martinez, J., Martineau, T. "Rethinking human resources: an agenda for the millennium." Health Policy and Planning; 13(4): 345-358. Oxford University Press (1998)

5. Tulenko, K., Møgedal, S., Afzal, M. M., Frymus, D., Oshin, A., Pate, M., Quain, E., Pinel, A., Wyndg, S., and Zodpeyh, S. "Community health workers for universal health-care coverage: from fragmentation to synergy." Bulletin of the World Health Organization (2013) http://www.who.int/bulletin/volumes/91/11/13-118745/en/

6. Serçe, F. C., Swigger, K. M., Alpaslan, F. N., Brazile, R.P., Dafoulas, G. A., Lopez-Cabrera, V. "Online collaboration: Collaborative behavior patterns and factors affecting globally distributed team performance." Computers in Human Behavior 27(1): 490-503 (2011) 